\title{
The Contribution and Application of Implantable and Wearable Devices on Animals
}

\author{
Yunshang Tian ${ }^{1, *}$ \\ College Information: Biology Department, University of York, York, North Yorkshire, YO10 5DD, UK \\ ${ }^{*}$ Corresponding author. Email: yt1031@york.ac.uk
}

\begin{abstract}
Implantable and wearable devices are commonly used on the human body. Such as artificial pacemakers, knee replacement, blood sugar monitors, apple watches. These devices greatly contributed to human life not only keep monitoring their body condition but also expend human life span. They also got developed in applying on the animal body. This paper will demonstrate how contributive implantable and wearable devices on animals. These devices help to position the location of animal that human can find their losing pet and leave some way for animal migration before they jammed in the urban area which may cause causality. They also raise the life span and save the lives of animals, especially wild animals to maintain the operation of the natural environment. Monitoring the body condition of an animal especially to those on the farm will be able to prevent and stop any outbreak of disease. These devices can also maintain the high production of animal husbandry. It is ensured that a well-developed technology not only is applied to the human body but also to animals because it will ultimately benefit our mother nature which is a win-win situation.
\end{abstract}

Keywords: implantable, wearable devices, track, Medical Treatment, Monitor

\section{INTRODUCTION}

Wearing smartwatches, such as Apples Watches and Intel MICA, has started becoming more popular and mainstream. Because they conduct lots of functions that are not limited to displaying time and temperature, but also the tracing function for humans' health and sports condition. In fact, the usage of trackers already emerged in the animal kingdom far before being promoted to humans. The application of these wearable trackers contributing to tracking speed, living habits, existence, and procreation of animals. There are more products that are specifically for animals. For example, a motion sensor and a removable heart simulator is formed together. When pets getting close to them, heartbeats will emerge from their body. These heartbeats will soothe the emotion of pets to help pets fall asleep and avoid any loneliness from them [1].

Although a wide variety of these theories are covered by literature, the review will focus on three key areas which will emerge frequently throughout the literature reviewed. Firstly, track and trace systems contribute to urban planning, filming of documentaries, research of animal behaviour, social behaviour, race, and reproduction. Secondly, in medical treatment, these trackers and external devices contribute to protecting endangered species, extending the life span, living standard and reproduction rate of animals. Lastly, the application of trackers to animals will be able to monitor the heartbeat, body temperature, eating situation of animals. This will contribute to the development of the aquaculture industry by conducting epidemic prevention. Although the literature presents these three areas in a variety of contexts, this paper will primarily focus on the application and contribution of implantable and wearable devices on animals.

\section{WEARABLE DEVICES TO TRACK AND TRACE ANIMALS}

With the application of these implantable and wearable devices on the body of animals, humans can track and realise the position of animals. The owner can understand more about the habit of their pets when applying those devices on their pet such as dogs and cats. These trackers can eliminate the gap between pet and owner because the owner may understand when their pet eat, what their pet love to eat when to sleep, when to walk their pet and when to play with pets. What is more important is that these trackers are able to find the position of the pet through the GPS. With the advanced technological development, the owner can easily find 
their trace and find their pet. Besides, the functions of these devices are extensive that they can eliminate any uncertainty. Virtual barriers and leashes are able to avoid pets running too far. The ultrasonic vibrational function is can help training dogs. The micro-speaker of the tracker allows the owner to talk with their pet. The internal LED light function will light up at night and it will flash once the pet got to lose [7].

Furthermore, these devices make a great contribution to saving wild animals and endangered animals. After applying them to a wild animal, it will be more convenient for the researcher to not only tracing the movement trajectory of animals but also understand animal behaviour. It may help to predict their survival rate and situation of reproduction. For example, sea turtles only are active on the beach that is close to their lair. But those trackers contributed a lot because it helps the researcher to grasp the information of migration and how turtles know the foraging grounds. Before researchers and scientists implant trackers on sea turtles, they need to process the turtle's shell through measuring, scrubbing, and rubbing it. Next, they will stick a transmitter on the turtle's shell. This substance of the transmitter is similar to glue which is a substance that can be used for human's teeth mold. After all, the epoxy adhesive will be used to cover the transmitter. Meanwhile, the transmitter contains a satellite tag and retractable antenna transmitter that is convenient for turtles to drill into smaller places. When every time a turtle swim to the sea surface, there will a transmutation from the satellite tag to a space satellite. After that, it will be transmitted to the researcher's computer on the ground via the satellite. Furthermore, mastering the positioning information of migratory animals is able to keep the distance between human and wild animal [6].

In June 2021[13], a group of wild elephants needed to change their habitat by passing through the urban are in Yunnan in China. This situation has never happened before. Relevant departments apply the positioning systems to analyze the life habits of wild elephants in order to predict their movements. The road was opened in advance to evacuate inhabitants, so that there were no property loss and casualties. Therefore, tracker not only contribute to pet and the owner, but also the whole ecosystem that wild animal can be protected with wellorganized system by scientists.

\section{CONTRIBUTIONS AND APPLICATIONS IN MEDICINE TREATMENT}

The application of these implantable and wearable devices on the animal body is greatly convenient for the therapy and recovery of the animal during veterinary work. Because it not only helps shorten the time of diagnosis and treatment but also lower the cost of the therapy. According to Granger's experiments (2020), he confirmed that electrical stimulation can help to restore bladder and bowel function of dog which has serious spinal cord injuries in an accident. Meanwhile, artificial lungs are developed in helping breathe sheep [3]. Hence, the development of medical treatment can improve the quality of life of animals and also extend their lifespan, thereby continue the existence of endangered species.

A dolphin was bound in the crab trap, she got caught, causing her tail to necrotize and fall off [8]. The tail of dolphins not only can maintain balance but also provide momentum. If the injured tail is not treated as soon as possible, the lower half of the body will deform, and the life span will be greatly reduced over time. Winter was subsequently sent to the Marine Aquarium in Clearwater, where it is housed for 14 years. Winter caused the loss of its tail to swim awkwardly, with its tail turning up and downside by side. In turn, Winter was equipped with a plastic silicone tail that would typically enable it to swim and even backflips. In addition, search and rescue dogs are vulnerable and easy to face any amputations at work. To provide a better life for the injured dog, a prosthesis can provide them with the ability to move after the surgery. Hence, if the mechanical exoskeleton can be put into use, search and rescue dogs may continue to work because it allows dogs to have motion support. Due to the limitation of resources, certain equipment and material are insufficient for the medical treatment of humans. Hence, they can be used for endangered species and animals with more contribution preferentially, such as search and rescue dogs and Chief Mouser.

\section{MONITOR THE SURROUNDING ENVIRONMENT AND PHYSICAL CONDITION OF ANIMALS}

The application of these devices is able to monitor the status of animals in all the farms to maintain their production rate. These devices generally lower the difficulties in detecting animal diseases and thereby lower the cost of aquaculture and reduced any anthropic risks. In commercial farms, it is common that sows often crush and kill piglets with their huge body. According to the statistics, around 1 out of 10 piglets is crushed and killed by the sow. In organic farms, these accidents happen more frequently which caused an average annual loss of 700,000 U.S. dollars and an aggregate annual loss of the entire industry is 8.9 billion U.S. dollars. To reduce the death rate of piglets, SwineTech is produced to be put on the body of sows. It can detect and measure the sound of pig and able to separate the special screams caused by suffocation from numerous sounds of piglet accurately. When the equipment of farrowing pen confirmed that the sows are squeezing a piglet, the electronic device on the sow will emit electromagnetic impulses to the sows to avoid any squeezing behaviour accidentally [9].

Moreover, there is an exclusive wireless ear tag for pigs on a farm [10]. After the installation of the ear tag, 
the body temperature, physical signs and mating cycle data of the pig will be fed back to its terminal's medical monitoring system which can be named as "electronic doctor" in real-time. This system applies Artificial Intelligent (AI) model to monitor and display the heat period, this helps the farmer to decide the best time for breeding. The AI model provides accurate and objective data which is far more accurate than the traditional manual testing method. Meanwhile, this system can also provide a warning before the disease happen to avoid a large outbreak of disease that cannot be noticed through human eyes. These real-time data collected by smart ear tags can be sold to insurance companies and banks to improve the financial situation of the entire animal husbandry gradually. When smart ear tags are widely promoted to the farmers. The government and supermarkets can estimate the supply of pigs to the pork market and control the price of pork accurately according to the data.

FarmoteColor is developed and produced by a Japanese firm [11]. It is an electronic wearable device that can be stick to the cow's head to monitor their physical conditions in real-time. Then, these data and information will be analysed through the supporting software. Farmnote will notice the farmer through sending them a message once the result of the analysis shows that a cow is suffering from illness, ovulating or birth. Therefore, the Farmer can respond as fast as they can to solve those issues. Besides, Farmer can also integrate the information and data to the system of Farmnote to further improve the accuracy of the data analysis to the vital signs of the cattle. The scheme of this software and hardware system can greatly contribute to dairy cattle and beef. FarmnoteColor has successfully applied this system to 160,000 cows. Hence this electronic device has contributed a lot to animal husbandry and provide a stable food supply to humans.

\section{CONCLUSION}

It is no doubt that these implantable and wearable devices are greatly contributive to pet and wild animals. There are more similar devices with other functions and more advanced one are still in the testing stage of research and development. For example, a headset is developed by a researching team for dogs [12]. The headset can read the brainwave signals of a dog and play it in human language after analysing it through the internal control system. Once this device is put into use, some animal behaviour issues will be solved and understand easily because the communication between animals and humans is improved. It will further facilitate the rescuing operation and advanced protection to the wild animal. With the advancement of technology, there will be smaller, softer, and smarter devices that can be developed in the future. They may better be integrated with animals that these devices will be comfortable and not look any different as before. Moreover, if more sensitive monitoring devices are developed, they may be the best alarm before the potential outbreak of infectious diseases. Maybe these devices may detect the super disease like COVID-19 before it spread to other regions, so that government may have enough time to react to it at the initial stage. The development of these devices will both contribute to the animal and human world in reducing huge casualties and property loss. The development of human industry has destroyed many natural environments and animal habitats. The development of science and technology should also benefit to animals to facilitate harmony between humans and mother nature. This may be the key for long-term development in the future.

\section{AUTHORS' CONTRIBUTIONS}

This paper is independently by the author.

\section{ACKNOWLEDGEMENTS}

During the writing process, Andreas Demosthenous and Zhang Minglong provide me with a lot of help. Mr. Demosthenous not only taught me a lot about wearable medical devices, but also helped me find references to support my arguments. I learned from Mr. Zhang the working principle of the physics and electricity of wearable devices. Their suggestions and help are very helpful for me to organize my writing ideas. Thanks again.

\section{REFERENCES}

[1] Snowdon, C. T. and Teie, D. (2013). Emotional communication in monkeys: music to their ears? The evolution of emotional communication: From sounds in nonhuman mammals to speech and music in man, 24, pp.133-151.

[2] Macdonald, A., Hawkes, L. A. and Corrigan, D. K. (2021). Recent advances in biomedical, biosensor and clinical measurement devices for use in humans and the potential application of these technologies for the study of physiology and disease in wild animals. Philosophical transactions of the Royal Society of London. Series B, Biological sciences. [Online]. Available https://strathprints.strath.ac.uk/76586/.

[3] Zhou, K. et al. (2013). Biocompatibility Assessment of a Long-Term Wearable Artificial Pump-Lung in Sheep. Artificial Organs, 37 (8), pp.678-688. [Online]. Available at: doi:10.1111/aor.12049.

[4] Neethirajan, S. (2017). Recent advances in wearable sensors for animal health management. Sensing and Bio-Sensing Research, 12, pp.15-29. 
[5] Granger, N. et al. (2020). Bladder and Bowel Management in Dogs With Spinal Cord Injury. Frontiers in veterinary science, 7, p.583342.

[6] Sack, A. et al. (2017). MORBIDITY AND MORTALITY OF WILD TURTLES AT A NORTH CAROLINA WILDLIFE CLINIC: A 10YEAR RETROSPECTIVE. Journal of zoo and wildlife medicine: official publication of the American Association of Zoo Veterinarians, 48 (3), pp.716-724.

[7] Weiss, G. M. et al. (2013). WagTag: a dog collar accessory for monitoring canine activity levels. In: Proceedings of the 2013 ACM conference on Pervasive and ubiquitous computing adjunct publication. UbiComp '13 Adjunct. 8 September 2013. New York, NY, USA: Association for Computing Machinery. pp.405-414. [Accessed 16 July 2021].

[8] Sun, Q. et al. (2010). Structure and Mechanical Properties on Tail Flukes of Dolphin. Journal of Aero Aqua Bio-mechanisms, 1 (1), pp.45-50.

[9] Mumm, J. et al. (2018). Sow behavior, heart rate, and cortisol responses to a novel piglet crushing prevention technology to reduce pre-weaning mortality. Champaign, 96 (3), pp.12-13.

[10] Xiong, B. et al. (2012). Development on mobile traceability system of feeding process of pigs and quality safety of its meat products based on $3 \mathrm{G}$ technology. Transactions of the Chinese Society of Agricultural Engineering/Nongye Gongcheng Xuebao, 28 (15), pp.228-233.

[11] Matoba, S. et al. (2109). 162 Detection of ovulation disorders and normal ovulation using wireless sensors of ventral tail surface temperature and neck acceleration data in Japanese Black cows. Reproduction, fertility, and development, 32 (2), pp.207-208.

[12] Valentin, G. et al. (2015). Towards a canine-human communication system based on head gestures. In: Proceedings of the 12th International Conference on Advances in Computer Entertainment Technology. ACE '15 65. 16 November 2015. New York, NY, USA: Association for Computing Machinery. pp.19. [Accessed 16 July 2021].

[13] BBC News. 2021. China elephants: Herd on mammoth $500 \mathrm{~km}$ trek reaches Kunming. Arqdqh \# Available at: $<$ https://www.bbc.co.uk/news/worldasia-china-57348255 $>$ [Accessed 15 July 2021]. 